\title{
Simple Guidelines for Authors: Open Journal of Mathematics and Physics [1]
}

\author{
Open Collaboration*† \\ October 17, 2020
}

\begin{abstract}
Simple guidelines are presented for authors willing to publish their original work in the Open Journal of Mathematics and Physics (0JMP) [2].
\end{abstract}

keywords: open science, diamond open access, microarticles

The most updated version of this paper is available at https://osf.io/fk836/download

\section{Introduction}

1. Paywall Journals are harmful to free open science in many ways [3].

2. Diamond Open Science provides a free publishing system both for authors and for readers.

3. OJMP is a Diamond Open Science Journal that publishes microarticles [4].

*All authors with their affiliations appear at the end of this paper.

${ }^{\dagger}$ Corresponding author: mplobo@uft.edu.br| Join the Open Collaboration 


\section{Diamond Open Access}

4. Authors publish articles for free.

5. Readers access and read articles for free.

\section{Overlay Journal}

6. OJMP is an Overlay Journal [5,6].

7. Here [7], you can find some guidelines on how to build your own highquality overlay journal [8].

\section{Open Invitation for New Collaborators}

8. All articles published in the OJMP can be updated at any time.

9. Important: Authors must accept new co-authors as long as the collaborators send at least one substantial progress for their published work.

\section{Types of Articles}

10. Conjecture: A conjecture that connects at least two well established scientific results [9].

11. Draft: An original piece of work waiting for collaborative (open) peer review.

12. Knowledge Base: Results presented by tags [10].

13. Microresearch: Contains one original scientific result [11].

14. Microreview: A well established scientific result shown in an original perspective [12]. 
15. Original Idea: Presents original steps delineating a specific open problem [13].

16. Original Insight: Contains an original insight about a well established scientific result [14].

17. Pedagogical: Presents creative ways for learning and teaching Mathematics and Physics [15].

18. Pre-Requisites: Addresses the full list of pre-requisites needed to understand a given mathematical theorem or a physical theory [16].

19. Question: Sketch for an innovative scientific question.

20. White Paper: The methodology of a white paper applied to scientific articles.

\section{Articles can be updated after being published}

21. Probably the most profound and innovative quality of the microarticle publishing format, as described here [4], is the ability to update the paper unlimited times, even after it has been published.

22. All versions of the article remain public at the Open Science Framework preprint platform [17].

23. At each update, it is expected that the lead author send an email to all co-authors with a list of the changes made in the manuscript.

24. The order of co-authorship can be modified by the lead author.

\section{Benefits of becoming a co-author on OJMP}

25. OJMP offers a free and fast publishing system.

26. You can strengthen your network of scientific collaboration. 
27. Your curriculum is enhanced, you publish more papers, get more citations, and improve science being part of a collective intelligence.

\section{License}

28. Unless otherwise noted, the articles in the OJMP are licensed under a Creative Commons Attribution 4.0 International License [18].

\section{Copyright}

29. Authors of the articles published in the OJMP retain copyright.

30. The authors declare all their text and figures are free of plagiarism.

\section{About Retractions}

31. The following issues qualify for retraction:
(a) falsification of results;
(b) copyright violation;
(c) incorrect results not updated by the authors after the editor's notification.

32. In case of retraction, authors should remove the header from the first page of the paper and submit it to the preprint server as a new version under the original preprint link.

\section{Final Remarks}

33. We look forward to receive your original work written in a microarticle format to be considered for publishing in the OJMP [4]. 
34. In addition, we also encourage each citizen/scientist to run hers/his own Diamond Open Access Journal [5-8].

\section{Open Invitation}

Review, add content, and co-author this paper [4,19].

Join the Open Collaboration.

Send your contribution to mplobo@uft.edu.br.

\section{Open Science}

The latex file for this paper together with other supplementary files are available [20].

\section{Ethical conduct of research}

This original work was pre-registered under the OSF Preprints [17], please cite it accordingly [19]. This will ensure that researches are conducted with integrity and intellectual honesty at all times and by all means.

\section{Acknowledgement}

+ Center for Open Science https://cos.io

+ Open Science Framework https://osf.io 


\section{References}

[1] Lobo, Matheus P. (Chief Editor) Open Journal of Mathematics and Physics. OSF Preprints, 2019. https://ojmp.org

[2] Epstein, Richard Allen, and Richard Allen Epstein. Simple rules for a complex world. Harvard University Press, 2009.

[3] PAYWALL. The Business of Scholarship. 2019 https://paywall themovie.com/paywall

[4] Lobo, Matheus P. "Microarticles." OSF Preprints, 28 Oct. 2019. https://doi.org/10.31219/osf .io/ejrct

[5] Van Noorden, Richard. Mathematicians aim to take publishers out of publishing. Nature News, 2013.

https://www . nature. com/news/mathematicians-aim-to-takepublishers-out-of-publishing-1.12243

[6] Ball, Philip. "Leading mathematician launches arXiv 'overlay' journal." Nature News 526.7571 (2015): 146.

https://www . nature. com/news/leading-mathematicianlaunches-arxiv-overlay-journal-1.18351

[7] Lobo, Matheus P. "A Do-it-yourself Overlay Journal." OSF Preprints, 27 June 2019. https://doi .org/10.31219/osf .io/gy8qv

[8] Lobo, Matheus P. "Darwinian Natural Selection of Articles in Overlay Journals." OSF Preprints, 29 June 2019.

https://doi.org/10.31219/osf .io/z8e3m

[9] Lobo, Matheus P. "The Interior of a Black Hole and the Void of Spacetime." OSF Preprints, 12 May 2019.

https://doi.org/10.31219/osf .io/awfx8 
[10] Lobo, Matheus P. "Open Mathematics Knowledge Base." OSF Preprints, 13 May 2020.

https://doi.org/10.31219/osf .io/evq8a

[11] Lobo, Matheus P. "A Hole in the Black Hole." OSF Preprints, 18 Apr. 2019. https://doi.org/10.31219/osf.io/js7rf

[12] Lobo, Matheus P. "Gödel's Incompleteness Theorem in a Nutshell." OSF Preprints, 4 Sept. 2019.

https://doi.org/10.31219/osf .io/gphsk

[13] Lobo, Matheus P. "Time Travel: Coexistence of Past, Present, and Future?." OSF Preprints, 2 Sept. 2019.

https://doi.org/10.31219/osf .io/7ruay

[14] Lobo, Matheus P. "Cancer: Division by Zero." OSF Preprints, 20 Jan. 2020. https://doi.org/10.31219/osf .io/34uft

[15] Lobo, Matheus P. "Teacher as a Researcher in Pure Mathematics." OSF Preprints, 9 Dec. 2019.

https://doi.org/10.31219/osf .io/849gh

[16] Lobo, Matheus P. "Pre-requisites for Frobenius Theorem (in Real Division Algebras)." OSF Preprints, 15 June 2019.

https://doi.org/10.31219/osf .io/gv8js

[17] COS. Open Science Framework. https://osf .io

[18] CC. Creative Commons. Attribution 4.0 International (CC BY 4.0). 2019. https://creativecommons.org/licenses/by/4.0

[19] Lobo, Matheus P. "Simple Guidelines for Authors: Open Journal of Mathematics and Physics." OSF Preprints, 15 Nov. 2019.

https://doi.org/10.31219/osf .io/fk836

[20] Lobo, Matheus P. "Open Journal of Mathematics and Physics (OJMP)." OSF, 21 Apr. 2020.

https://doi.org/10.17605/osf.io/6hzyp 


\section{The Open Collaboration}

Matheus Pereira Lobo (lead author, mplobo@uft.edu.br) $)^{1,2}$ https://orcid.org/0000-0003-4554-1372

${ }^{1}$ Federal University of Tocantins (Brazil)

${ }^{2}$ Universidade Aberta (UAb, Portugal) 